\title{
Exploring Public Perceptions and Understanding of Dementia: Analysing Narratives from the Mass Observation Project
}

\author{
Veronica Olsen ${ }^{1}$, Lou Taylor ${ }^{1}$, Kirsty Whiteley ${ }^{1}$, Annie Ellerton ${ }^{1}$, Paul Kingston ${ }^{1,2}$, and \\ Jan Bailey 1 \\ ${ }^{1}$ Centre for Ageing and Mental Health, Faculty of Health and Social Care, University of Chester \\ ${ }^{2}$ Corresponding author: p.kingston@chester.ac.uk
}

\begin{abstract}
Over 850,000 people living in the United Kingdom have been diagnosed with dementia, yet knowledge about this condition amongst the general population remains relatively poor. Many studies have evaluated the level of public knowledge and understanding about dementia from a research and professional service perspective, however none have considered this condition from the perspective of the wider public. In this preliminary overview, we analyse and describe high level narratives collected from 143 respondents to a dementia Directive commissioned to the Mass Observation Project. These narratives present a perspective on the public knowledge and understanding about dementia not previously considered, where respondents have written openly about their own experiences, and reflected on their perception of the wider public's knowledge and understanding about dementia. This unique perspective importantly enhances our knowledge about the public's understanding and awareness of dementia, and informs the main areas of public concern found in the analysis: care responsibilities, impact on relationships, and fears about developing dementia.
\end{abstract}

\section{Key Words}

Dementia, Mass Observation Project, public perception, respondents, observers, public understanding, narratives.

\section{Introduction}

The World Health Organisation (WHO) describes dementia as

'a syndrome due to disease of the brain, usually of a progressive nature, in which there is a disturbance of multiple higher cortical functions including memory, thinking, orientation, comprehension, calculation, learning capacity, language and judgement; and impaired cognitive function'(World Health Organisation, 2012, p. 7).

The progressive decline in cognition associated with dementia is of sufficient severity to interfere with activities of daily living (Giebel, Sutcliffe, \& Challis, 2015; Knopman et al., 2001). 
To date, over 200 subtypes of dementia have been defined, each one with a different pathway or process (Stephan \& Brayne, 2010). Currently (2018), finding a cure or even effective drug treatments is proving elusive, with some researchers suggesting it has 'replaced cancer as the scourge of modern times' (Van Gorp \& Vercruysse, 2012; Zeilig, 2015, p. 15) and the 'most feared condition for the 55+ age group' (Live in Care Hub, 2017). Indeed, the search is so elusive the pharmaceutical company Pfizer has decided to halt research into treatments for Alzheimers disease (Dwyer, 2018).

In the United Kingdom (UK) there are 850,000 people living with dementia; this figure is expected to rise to more than two million by 2051 (Dementia Strategy (UK) 2017-2020). Recent reviews by Stokes, Combes, and Stokes (2015) and Cahill, Pierce, Werner, Darley, and Bobersky (2015) suggest that knowledge about dementia in the UK and Ireland is still relatively poor amongst the general population. 'The most common misconception suggested across a range of studies, is that dementia is a normal part of ageing and there is a lack of clarity about the point at which normal age-related memory loss problems become severe enough to indicate dementia' (Cahill et al., 2015, p. 274). Diagnoses communicated to the public often appear to leave family carers and patients confused due to use of euphemistic and evasive terminology (Stokes et al., 2015). Raising public awareness and knowledge about dementia is essential to alert individuals to seek advice promptly for early diagnosis and disease management, optimisation of care, and enhancing the quality of life of individuals who are diagnosed and their potential caregivers (Rimmer, Wojciechowska, \& Stave, 2005).

Evidence suggests that many carers find it difficult to plan for the future due to a range of uncertainties after a dementia prognosis (Aggarwal et al. 2003; Aminzadeh, Byszewski, Molnar, \& Eisner 2007). As the majority of individuals diagnosed with dementia live in the community are cared for by a spouse or relative (Quince, 2012; Rabins, 1998; Zarit, Gaugler, \& Jarrott, 1999), it is important to fully evaluate the public's understanding and awareness of dementia from a variety of perspectives to identify service areas which require improvement.

Several international studies have examined public perception and knowledge of dementia; however a large extent of this work has focused on specific 'at-risk populations (first-degree relatives) making it difficult to generalise findings to the wider general public (Roberts, McLaughlin, \& Connell, 2014). Other researchers have assessed public understanding of Alzheimer's disease or dementia using structured 'knowledge tests' (Dieckmann, Zarit, Zarit, \& Gatz, 1988), surveys and questionnaires; some adapting existing tests to meet current needs (Anderson, Day, Beard, Reed, \& Wu, 2009; Arai, Arai, \& Zarit, 2008; Carpenter, Balsis, Otilingam, Hanson, \& Gatz, 2009; Roberts et al., 2014; Steckenrider, 1993). International research exploring the public understanding of dementia has revealed gaps and 
misconceptions on a global scale (Anderson et al., 2009; Cahill et al., 2015; Chung, 2000; Steckenrider, 1993; Sullivan, Muscat, \& Mulgrew, 2007).

'There is clearly a need for greater public awareness' (Yeo, Horan, Jones, \& Pendleton, 2007, p. 371) as without adequate information, family carers in all communities are 'likely to use existing lay knowledge to inform care planning' (Stokes et al., 2015, p. 221).

The existing literature therefore contains studies of public knowledge and understanding about dementia from a research and professional service perspective by use of more traditional data collection methods, however there is no evidence where the public have been asked to write openly about their own level of understanding about dementia, nor reflect on their perception of the wider public's understanding. These perspectives are essential to gain a wider appreciation of society's understanding and knowledge about dementia. To address this gap in knowledge, we pursued an alternative approach and analysed narratives collected from a sample of the UK general public who participate in the Mass Observation Project ${ }^{1}$. These narratives recognise the voice of the public as a vital resource to further evaluate the level of understanding and awareness of dementia in the UK.

\section{The Mass Observation Project}

The Mass Observation Project was founded in the UK in 1937 with the aim of collecting data on a wide range of social issues directly from the general public. This unique strategy has a panel of 500 members of the public ('Observers') who represent the 'ordinary people in Britain'. Observers are asked to respond, in writing, to a set of questions ('Directives') three times each year. Each Directive usually contains three questions; the Centre for Ageing Studies commissioned a Directive on dementia in Spring 2015. Observers are not given a specified word length for responses, which may be handwritten or electronic. Observers are free to select the Directives or part of the Directive to which they respond, and are asked to write unreservedly on any of their chosen topics. Anonymity is provided via a Mass Observation code, and Directive instructions advise observers not to inadvertently identify themselves or other people in their responses. Due to the complete anonymity of observers and the opportunity and freedom to write openly, this rich data source offers a unique insight into a representative sample of the views and perceptions of this populace. Furthermore, these narratives provide a level of candidness not frequently achieved through interviews.

The use of narratives has become a legitimate area of study for health care over the past 1020 years and service user perspectives of health conditions provide additional value alongside empirical studies (Benbow and Kingston (2016). Furthermore, Benbow and Kingston (2016) argue that narratives offer a model for learning, highlighting that service user' experiences are

\footnotetext{
${ }^{1}$ http://www.massobs.org.uk/
} 
a source of content and process knowledge that can be incorporated into service design and improvement.

The review conducted by Cahill et al. (2015) found very few studies adopted qualitative methods to evaluate public knowledge of dementia. They propose more qualitative research is needed to consider the complexities of, and contradictions in, the general public's knowledge and understandings of dementia. We have employed a qualitative approach to describe and analyse the narratives collected from individuals who responded to the Mass Observation Dementia Directive. The Directive asked observers to describe any experiences and memories of dementia identified in friends or relatives. Observers were also asked to respond to a series of brief questions about dementia which sought insight into perceived changes in knowledge and understanding about dementia over the past five to ten years, information on how knowledge has been acquired, and individuals' concerns about potentially receiving a diagnosis of dementia. Analysis of these narratives enabled us to ascertain the current level of understanding of dementia from a cross-section of the UK population. Furthermore, by asking observers to describe how they perceive the current level of knowledge and understanding about dementia by the wider public, we have the unique opportunity to consider the Directive from a peer-to-peer perspective.

This study presents a perspective on the level of public understanding of dementia not previously considered, and adds to the existing knowledge from a research and medical/care professionals' perspective of public awareness and the understanding of dementia.

\section{Demographics}

From the 500 observers in the Mass Observation Project panel, 143 responded to the Dementia Directive; for the purposes of this paper, we refer to these individuals as 'respondents'. Although predominantly white middle-class, respondents were from a wide demographic in terms of geographical location across England and Wales, occupation and marital status. Respondents represented both genders across a broad range of ages (Table 1) with a mean age of 59.1 for males and 59.4 for females.

\section{Table 1: Participant Age and Gender Details}

\begin{tabular}{|c|c|c|}
\hline Age & Male & Female \\
\hline$<25$ & 0 & 5 \\
\hline $26-35$ & 1 & 11 \\
\hline $36-45$ & 11 & 16 \\
\hline $46-55$ & 8 & 9 \\
\hline $56-65$ & 4 & 15 \\
\hline $66-75$ & 9 & 22 \\
\hline $76-85$ & 5 & 20 \\
\hline
\end{tabular}




\begin{tabular}{|c|c|c|}
\hline$>85$ & 2 & 3 \\
\hline $\begin{array}{c}\text { Not } \\
\text { specified }\end{array}$ & 1 & 0 \\
\hline Total & $41^{*}$ & $101^{*}$ \\
\hline
\end{tabular}

Almost half the respondents were retired; the others from a wide range of professions and studentships. More than half the respondents were married; the remaining individuals were single, widowed, civil partnership, divorced or cohabiting. Approximately $20 \%$ of respondents were from the south east of the UK; the other observers dispersed throughout England, Wales and Scotland. One respondent had been recently diagnosed with vascular dementia, and three others expressed real concerns of early stage dementia believing they had experienced symptoms.

\section{Methods}

We commissioned a Mass Observation Directive in Spring 2015 posing a range of questions about observers' knowledge and experiences of dementia. This paper reports the resulting data that is related to the questions listed in Table 2.

Table 2: Dementia Directive questions addressed in this paper

Do you have any memories of friends or relatives who had what we now
understand to be dementia?
What is your understanding of dementia and how it affects individuals?
Where did you gather this knowledge from?
Do you have concerns about experiencing dementia in the future?
How far do you think the general public are aware of dementia and
understand the condition? How do you think this has changed over the
past five to ten years?

To ensure complete anonymity for publication, each identification number issued to individuals upon registering with the Mass Observation Project, was assigned a second randomised number (R1 - R143) prior to our analysis.

Qualitative analysis software (QSR International Pty Ltd., 2017) was used to manage the data and facilitate analysis; word processed responses were uploaded directly to the programme and handwritten responses were scanned then uploaded.

A random sample of 10 narratives was read by all authors to familiarise with the data and establish initial themes. The aim of this paper is to therefore offer a 'high level first cut' given 
the productiveness of the data. Further papers are in production offering further sub-analysis of the key themes found in this first 'cut'.

All 143 narratives were then read and coded by three researchers. Themes were discussed and restructured into main and subthemes to provide an overview of the findings for this publication.

\section{Findings}

The 143 responses (72 typed and 71 handwritten) received from the Dementia Directive ranged from one sentence to multiple pages. Narratives were invariably candid, and often shared highly emotive and intimate details of experiences. We acknowledge some example quotations chosen for publication contain blunt and upsetting descriptions and language; however, we believe they effectively illustrate the fundamental findings from this study and epitomise the respondent's voice. The main themes and subthemes that emerged from the analysis are listed in Table 3.

Table 3: Main and Subthemes related to Dementia Directive

\begin{tabular}{|l|l|}
\hline Main Theme & Subtheme \\
\hline \multirow{2}{*}{ Understanding of dementia } & Disease symptoms \\
\cline { 2 - 2 } & Altered behaviours \\
\hline \multirow{2}{*}{ Impact on relationships } & Care responsibilities \\
\cline { 2 - 2 } & Impact on relationships and family dynamics \\
\hline & \\
\hline \multirow{2}{*}{ Stigma of dementia } & Fear of Dementia \\
\cline { 2 - 2 } & Changes in public perception \\
\hline
\end{tabular}

Whilst analysing the respondents' level of understanding about dementia, it became apparent there was a striking dichotomy between individuals with no direct experience of dementia $(28 \%)$ and those with family or friends living with dementia (72\%). Respondents with direct experience were mostly a relative of a person with dementia (Table 4). We reflect upon the pattern of frequent dichotomy (and sometime variation) between respondents with and without direct experience of care in dementia.

Table 4: Relationship of person with dementia to respondent with direct experience

\begin{tabular}{|l|c|}
\hline Relationship & $\%$ \\
\hline Parent/Parent in-law & 27 \\
\hline Other relative (Aunt, Uncle) & 24 \\
\hline Grandparent & 23 \\
\hline Friend & 10 \\
\hline Neighbour & 7 \\
\hline
\end{tabular}




\begin{tabular}{|l|c|}
\hline Work Colleague & 6 \\
\hline Spouse/Partner & 2.2 \\
\hline Son/Daughter & 0.8 \\
\hline
\end{tabular}

\section{Understanding of Dementia}

\section{Disease Symptoms}

Loss of memory was cited by sixty (42\%) respondents as the main symptom of dementia; most respondents understood dementia as initial short-term memory loss, and enduring long-term memory. Generally, respondents with little direct experience of dementia tended to have a reasonable, but more rudimentary understanding of dementia; descriptions were often limited to explaining the more commonly known effects such as memory loss. In contrast, respondents who had direct experience of close family or friends affected by dementia had a deeper level of understanding of the symptoms beyond memory loss, and gave more emotive and personal insights into the impact dementia has on daily life. These respondents also perceived the degree of public understanding about dementia symptoms was mostly influenced by the level of experience; a number of these respondents remarked the impact of memory loss was understated by those who had minimal experience with a person with dementia:

The implications of the memory loss are perhaps more far-reaching than people perceive (R3: Female aged 40).

Despite this observation, many respondents reflected that personal experience did not always enable them to fully explain the effects of dementia to others with less familiarity of the disease:

It is often difficult to explain to others when they ask how grandma is - how long someone can live and decline with this illness but still be so physically well as she is. (R2: Female aged 30).

A number of older respondents suggested reasons for perceived lack of public understanding and awareness about dementia included limited access to information on the disease effects and mechanism, and a reluctance to speak openly. These older respondents reflected that they and their peers had previously viewed dementia-like symptoms as something that happened with normal ageing:

In the past I think it was accepted as normal that 'old age' produced a change in character and in the brain, a decline in mental and physical capabilities, forgetfulness, memory loss,

difficulties with expressing oneself, etc. was all part of getting older (R124: Female aged 82).

\section{Altered Behaviour}

Respondents with direct experience of dementia described symptoms of altered behaviours such as forgetfulness, paranoia and aggression: 
One of the side effects of the disease that I witnessed was the anger of the sufferer, in fact I know that my father was quite violent towards my mother, which was completely opposite of what he had been like. (R93: Female aged 68).

These respondents frequently reflected on the challenge of providing care to people with altered behaviours, and acknowledged the constant changes to care needs required with disease progression. Many individuals insisted family could provide the best emotional support and stability, including the least disruption to the person with dementia, in the early stages of disease progression. Contrariwise, they also realised there would be limitations to long-term family care provisions as symptoms became more challenging. A clear difference in level of understanding was evident in respondents with less experience however, who rarely commented on care requirements; many not considering the ramifications of altered behaviour beyond the early stages of memory loss.

\section{Impact on Relationships}

\section{Care Responsibilities}

Typically, respondents with minimal experience had a limited understanding with regard to the reality of caring for someone with dementia and suggested more idealistic approaches to care:

Ideally, someone with dementia should be cared for by someone in their family as far as possible, I cannot imagine anything worse than not being with people you know and trust, and in your familiar surroundings (R26: Male aged 48).

Other respondents expressed high moral aspirations should the need to provide dementia care arise; although some appeared to lack a deeper insight into what would be involved:

I would want any friend of mine who had dementia to be treated with respect and sympathy... to have someone constantly near them, smiling and tolerant and encouraging (R121: Female aged 65).

Individuals with experience of providing dementia care, or who had a close friendship with a carer, described the demands on carers' emotional and physical health, and the difficulties experienced in adjusting to a continually changing relationship dynamic:

A friend cared for her Mum at home for a number of years. She sacrificed her career, her life, and it nearly killed her. Eventually she became too ill and overwrought to do it (R64: Female aged 86).

Overall, many respondents expressed concerns over their own limited skills for providing suitable dementia care following a diagnosis, even in the early stages. Some individuals recognised care can involve a range of tasks, including looking after someone with short-term memory loss who 'poses a threat to herself by forgetting to turn off her gas hob' (R31: Male 
aged 49) through to someone 'suffering from hallucinations' (R31: Male aged 49). Not all respondents believed they were capable of taking on a caregiver role, whilst others were unwilling to be the caregiver due to the nature of their former relationship with the person. Some respondents dreaded a second experience of providing care:

I would make a lousy full-time carer. It just isn't me. l've [had] that experience in my teens and I won't do it again (R47: Female 50).

\section{Impact on Relationships and Family Dynamics}

More than 90 (>63\%) respondents made reference to the emotional impact that dementia can have on relationships. Individuals with less experience wrote very little about impact, however those with direct experience described the tremendous strain on relationships alongside feelings of guilt, frustration and grief. The reality of the strain was quite evident for this respondent:

Without any support you are imprisoned within a cage of responsibility (R66: Male 45).

A number of individuals with direct experience of care described occurrences of entire families worn out by distress, worry and unrelenting responsibility, some judging it to be 'a physical risk to sufferer and carer' (R51: Female aged 64). Other respondents reflected on the additional strain caused to other personal relationships, with one individual referring to the breakdown of their own marriage whilst caring for a parent with dementia. The tension experienced between family members who did not share a similar empathy towards a relative with dementia was also described:

My mother, aunts and uncle are not blessed with patience, so when [my grandmother] is explaining something or telling a story, she often gets mixed up, forgets what she has said, or takes a while to say what she is saying. There is also a lot of eye rolling or laughing at what she has said, which I don't appreciate (R59: Female aged 25).

Respondents with direct experience of providing dementia care overwhelmingly considered that family care in the latter stages of dementia was unsustainable, citing inexorable emotional, financial and physical strain. These respondents also described feelings of guilt at locating loved ones into care facilities for the latter stages of the disease.

Several respondents with caring experience provided powerful insight into the inevitable change in relationship dynamics as an individual's dementia symptoms progressed:

It has been very painful for her, as an only daughter, trying to care for her mother, without losing her temper at some of the things the mother has done (R79: Female aged 56).

Respondents also reflected on how caring for a person with dementia had changed the hierarchical structure and dynamics within families: 
My granny's relationship with all her family changed over time. She went from being the family matriarch to a child. Her children and grandchildren had to slowly adjust from being the cared for to providing care (R71: Female aged 39).

Other respondents with experience in providing care noted family members with dementia often experienced confusion about family relationships and identity. Disorientation between individuals within a family were a common theme, for example mistaking grandchildren for their own children. Several respondents experienced in caring for people with latter stage dementia believed the onlookers experienced the most distress, suggesting the person with dementia was often unaware of their condition:

Dementia is tragic, not so much for the sufferer who probably has little idea of what is happening, but for the family. It is so sad to see the gradual deterioration of a loved one (R126: Male aged 78).

\section{Stigma of Dementia}

\section{Fear of Dementia}

When asked to describe their personal concerns about developing dementia, respondents generally divided into those who were actively worried and those who were unconcerned. Respondents with direct experience of dementia tended to have greater concerns about developing dementia than those with less experience, and were unsurprisingly disturbed by the prospect of a diagnosis:

This loss of memory, identity, [and] control of your life frightens my peer group more than anything (R143: Female aged 79).

Individuals with anxieties singled out specific concerns including the disease itself, fear of its impact on relationships, apprehension over standard of care and responsibility of care delivery, amongst others. This awareness led them to contemplate a potentially frightening future, with many stating they feared dementia more than any other disease:

I fear dementia more than cancer (R22: Female aged 62).

In contrast, approximately $20 \%$ of the respondents stated they were genuinely not concerned about developing dementia, citing a number of reasons for this stance. Some of the older respondents in this group, for example septuagenarians and octogenarians, judged they had managed to reach this age in good health and perhaps had no need to be anxious.

A high proportion of the unconcerned respondents were under 50 years of age despite the growing awareness that dementia can affect younger people. These respondents considered themselves too young to worry about the possibility; the future held promise of medical 
advances in treatments or cure. Their confidence increased if they considered they lived a healthy and balanced lifestyle and relatives had not been diagnosed with dementia.

Interestingly a small number of respondents stated they intentionally avoid thinking about the possibility of developing dementia:

I would prefer not to have such acute knowledge of illness (R15: Female aged 80).

\section{Changes in Public Perception}

Respondents were asked to reflect on how they perceived the wider public's knowledge and level of understanding about dementia. Approximately $30 \%$ of respondents perceived there had been an increase in public understanding and awareness of dementia over the past five to ten years, and improved tolerance and empathy towards those experiencing dementia. $A$ number of respondents suggested the increased public awareness was due to more knowledge about the ageing population and longer life expectancy gathered from media, or by knowing someone who had been diagnosed.

In contrast, some respondents suggested there had been little change in the public's awareness and understanding of dementia, and it had not developed beyond a basic level:

It's hard to say how the general public view dementia but, based on my own experience, there is a lot of ignorance about it. As I said, I think most people associate it with memory loss in old people, which is one very small part of it (R120: Female aged 22).

Many respondents recognised an increased public awareness of dementia diagnosis in younger people, although one respondent reported a perceived reluctance by friends to accept her own dementia diagnoses, despite obvious changes in behaviour:

I encounter a degree of ignorance around my memory, short-term memory function is poor, but my episodic/long-term memory is still working perfectly so people say 'you've not got dementia at all, you remembered our school outings/birthday parties etc.' and 'oh everyone forgets where their keys are and stuff, there's nothing wrong with you' (R43: Female aged 38).

Older respondents vividly recounted memories of historically accepted treatment for conditions now known as dementia; isolation from society and the shame and stigma associated with mental health illness was commonplace earlier in their lifetime:

I knew well that there were such places as mental hospitals and lunatic asylums - grim places they were too - and if mentioned at all they were spoken of in low tones. I wouldn't say that people were more callous then but such was the shame, the ignorance and superstition attached to mental illnesses... The word 'dementia', I am pretty sure, would have been an unfamiliar word to the people of that era (R97: Male 93 years). 
Respondents perceived the wider public had become somewhat more aware and tolerant of conditions such as dementia in the past five to ten years, helped particularly by high profile/celebrity exposure (sharing their own dementia experiences) in the media, followed by more information through various media (TV, radio, print media, movies, internet):

I think people are more sympathetic to the condition due to either family members suffering or from gaining knowledge where people have been more open in the media and discussing their condition (R8: Female 39).

Despite the improvements in public awareness and tolerance of dementia, most respondents who had experience in providing dementia care agreed that, although some care tasks in the early stages were manageable, they could not provide an adequate level of care in the latter stages of the disease. Members of the public without carer experience were perceived by many respondents to have a limited understanding about the impact providing dementia care has on family relationships. The totality of caring challenges and the perceived lack of understanding in peers without carer experience is captured in this response:

Until you have something to do with a person who has dementia, you cannot comprehend the sheer frustration and loneliness it involves, for all parties (R136: Female aged 46).

\section{Discussion}

\section{The Use of Narratives}

The Centre for Ageing Studies commissioned a Dementia Directive from the Mass Observation Project in Spring 2015 to gain a better appreciation of the public's knowledge and understanding of dementia, by collecting narratives. The use of narratives provided an opportunity for respondents to describe the level of knowledge and understanding about dementia, and highlighted areas that may require more attention to improve the experiences of people living with dementia, their carers, and the wider public. The Directive was also used to establish how respondents perceived the wider public's level of knowledge and understanding of dementia from a peer-to-peer perspective. The need for more research on the general public's understanding has previously been highlighted (Langdon, Eagle, and Warner (2007), and Rimmer et al. (2005) have called for more comprehensive political, educational and social strategies to raise public knowledge of dementia to enhance the quality of life of people diagnosed and their caregivers. Narratives in this Directive have added further evidence to a number of studies (for example, Cahill et al., 2015; Kenigsberg et al., 2016; Milne, 2010; Quince, 2012; Zarit et al., 1999) that suggest particular attention is needed to better manage the delivery of care at all stages of the disease. Furthermore, Benbow and Kingston (2016) postulate that carer narratives may be an important consideration in order to enhance service provision, and Zeilig (2015, p. 13) suggests there is increasing recognition within academic circles of the importance of exploring stories about dementia through 
narratives, particularly to 'help uncover truths about how a society imagines and engages with people who live with dementia'.

The narratives in this study offered a unique and progressive perspective; a number of respondents gave astonishingly comprehensive and personal accounts of their experiences. Many narratives were several pages long, and read as if this was the first opportunity to openly share experiences of painful memories; the benefit of anonymity was plain to see. Others wrote very short responses, either stating they had little knowledge or experience, or preferred not to write about a topic that was upsetting; one simply wrote 'Sorry, didn't feel like tackling part one' (R137: Female 43 years). We consider the Mass Observation narratives provided an insight into the public's opinions and experiences not achieved by other methods such as questionnaires or interviews; the unrestricted format and complete anonymity allowed respondents to share views in a more open format and provided a rich and informative data set.

\section{Respondent Understanding via Experience}

When reading the narratives, a clear distinction was evident between individuals with direct experience of dementia and individuals with limited or no exposure. A large number of respondents $(n=60)$ cited memory loss as the main symptom of dementia, these findings are consistent with Cahill et al. (2015), however respondents with direct experience demonstrated a more in depth understanding of dementia, usually gained by providing care or by insight via observation into the experiences of close friends involved in a carer role. Accounts from respondents with experience tended to be more emotive and described the impacts (beyond memory loss) on activities of daily living comparable to Giebel et al. (2015), however some respondents reflected that greater personal experience with providing dementia care did not always enable them to explain the full effects of the disease to peers. Narratives from respondents with less direct experience, whilst also using emotive words such as '...it must be frightening (R51: Female 64), to describe their understanding of dementia, offered less detail about symptoms beyond memory loss or forgetfulness.

\section{Responsibilities and Impact of Care Provision}

Individuals with carer responsibility listed forgetfulness, paranoia and aggression as common challenges experienced during disease progression, all of which demanded constant changes to care requirements. Several respondents reflected they felt unable to provide adequate care in the later stages of disease progression citing the increasing complexity and changing requirements as the main limitations. Others wrote about the intense physical and emotional aspects of providing care, whilst others conceded a lack of suitable skills. A study by Rimmer et al. (2005) echo similar impacts of providing dementia care; with carers describing the effects of this role as life changing (94\%), difficult (91\%), exhausting (89\%), demanding (86\%), and 
stressful (86\%). Respondents describing caring experiences also included extensive and moving details of how having a family member with dementia had impacted on relationships and altered family dynamics. These findings are consistent with Kenigsberg et al. (2016, p. 16), who describe the complex nature of dementia care 'involves multiple domains: functional, cognitive and intellectual, emotional, medical (including pain management), and end-of-life care'. Parkinson, Carr, Rushmer, and Abley (2016, p. 9) identify a 'burgeoning need to find a coherent approach to family carer support' and a 'notable absence' of literature that detail effective strategies to support family carers. Furthermore, Milne (2010) suggests a linking challenge whereby fragmented support for carers can impact on the carer experience; some studies (Betts Adams \& McClendon, 2006; Bowling \& Gabriel, 2007; Gethin-Jones, 2014) even propose that carers themselves are at risk of social isolation as a result of the dedication required.

Despite the literature recognising the complex nature of dementia care, many respondents with care experience expressed tremendous guilt at seeking professional care for a family member with dementia, even once symptoms have developed beyond their caring capabilities. In contrast, other respondents felt comfortable stating they were unwilling to provide care due to the nature of the former relationship with the person with dementia, and some claimed previous care experience had influenced their moral stance not to undertake a care role again. Of note, none of the respondents with experience of providing dementia care described their experience as positive, unlike some participants in the study by Rimmer et al. (2005), who reported some carers found their experience rewarding $(27 \%)$, fulfilling $(30 \%)$ or enriching (33\%). Generally, respondents in our study with experience in care provision, perceived an inability to comprehend the breadth of challenges faced in a carer role by those with less or no experience

\section{Changes in Public Perception and Reduction in Stigma}

Approximately $30 \%$ of respondents perceived there had been an increase in public understanding of dementia over the past five to ten years, citing reports about an ageing population and longer life expectancy, or by knowing someone who had been diagnosed as sources of information. Despite studies, for example Milne (2010), suggesting the public generally understand dementia as a normal and inevitable part of the ageing process, many respondents in this study perceived the level of public understanding had somewhat developed from this belief. Some older respondents who acknowledged that previous generations had accepted dementia symptoms as age-related behavioural changes perceived a greater understanding now existed. A number of respondents perceived that the public were increasingly aware that dementia could affect the younger population. Many respondents commented that an increase in awareness about dementia could be the result of high 
profile/celebrity exposure and a range of representations in film, TV, and books, and suggested these shared experiences had improved public tolerance and empathy towards those experiencing dementia.

Zeilig (2015, p. 14) reports that high profile figures with dementia who have 'started to speak without mediation' have had a positive impact on raising public awareness by 'representing and advocating themselves'. The importance of these public role models was emphasised by Milne (2010) who advises 'presenting positive images of people living well with dementia in the media could make a significant contribution to demystifying the condition and challenge negative attitudes that currently pervades public opinion'. Furthermore, Langdon et al. (2007, p. 998) encourages open conversation to increase awareness and suggests the 'avoidance of frank discussion may fuel the sense that dementia is a taboo subject. Many of our respondents reflected this in their narratives, one stating:

It does help knowing that dementia is increasingly common so that it can be discussed openly. Cancer was once unmentionable. It isn't now. And dementia has been brought into the light in recent years (R18: Male aged 71).

Curiously, one respondent perceived the increased awareness had been so successful that she believed the public were "worried well', swamping memory clinics in an effort to ensure they do not have dementia, making those who do need the clinics face longer waiting times for appointments' (R138: Female aged 47). Kim, Sargent-Cox, and Anstey (2015) also found fear was the main motivation for changing health behaviour and lifestyle to reduce the risk of developing dementia, and highlighted the importance of accurate public health education to motivate prevention through a positive and healthier lifestyle.

Despite the perceived increase in public tolerance and empathy expressed by the respondents, Langdon et al. (2007, p. 995) found that people diagnosed with dementia retain a reluctance to 'share their diagnosis outside of family for fear that the public would reach misguided conclusions about the implications of the diagnosis'. Langdon et al. (2007) also suggests the stigma of dementia may arise partially from the medical terminology; participants in that study expressed a strong aversion to the word 'dementia'. Many of our respondents shared this view; a number referred to the fear surrounding the term 'dementia'. A number stated they fear dementia more than any other disease; these findings align with a growing number of publications reporting this trend (Live in Care Live in Care Hub, 2017; Van Gorp \& Vercruysse, 2012; Zeilig, 2015). Generally, individuals who actively worried about developing dementia were more experienced in caring for a person with dementia; this finding is consistent with studies by Kim et al. (2015) and Williams, Tappen, Rosselli, Keane, and Newlin (2010) who report that experience of a relative with dementia increased personal vulnerability. In contrast, other respondents stated they were nonchalant, whilst some deliberately avoided 
the topic. Many respondents admitted their fear was due to a lack of personal understanding, including those who had direct experience of providing care. A number of respondents perceived there were a number of gaps in public general knowledge and understanding about dementia symptoms and care requirements. Many stated that due to increasing disease prevalence, there was a need for wider education to enable more effective care planning and reduce some of the fears held by the public.

\section{Limitations}

Limitations to this study are acknowledged. The Mass Observation respondents are predominantly from white middle-class backgrounds, and although they represent a wide demographic in terms of geographical location, occupation and marital status, this study is not representative of all socio-economic groups. Furthermore, it is acknowledged the respondents did not include a range of ethnic minority populations. Nielsen and Waldemar (2016) and Cahill et al. (2015) suggest ethnic minority populations remain underrepresented in dementia research and dementia care in terms of diagnosis, treatment, and use of social services, whilst others (for example, Parveen, Peltier, \& Oyebode, 2017) discuss the issues of cultural sensitivities that exist for further research.

Respondents represented both genders across a broad range of ages, however, two thirds of the respondents were female. Because Mass Observation panel respondents $(n=500)$ are permitted to reply to any of the Directives, it is likely that respondent populations may be unintentionally skewed towards topics of personal interest. Likewise, it is also entirely possible that observers may avoid responding to topics that they find difficult or upsetting.

Challenges were encountered in this study, such as difficult to read handwriting and interpreting the tone of some narratives. Although the narratives provided a rich data set, it would have been helpful to follow up with some respondents by interview to further explore their documented experiences; the anonymity of participants in the Mass Observation project does not permit this.

\section{Conclusion}

The Mass Observation narratives have provided us with a considerably enhanced knowledge of the public's understanding and awareness of dementia, and have highlighted the main areas of public concern around care responsibilities, impact on relationships, and fears about developing dementia. The opportunity for respondents to write without constraints of length, content or language has given a unique viewpoint to expand knowledge about the current level of public understanding and awareness of dementia. The findings of this study have complemented and enriched the existing literature by enabling insight through shared experiences. The importance of evaluating the perceived wider public's understanding of 
dementia from the respondents' viewpoint has also been argued. Furthermore, the research team have been privileged to read exceedingly personal and intimate accounts of dementia experiences from the perspective of respondents, unlikely to be achieved through other data collection methods.

Previous studies have evaluated public understanding and awareness using more traditional data collection methods, predominantly from a research and professional service perspective. It is suggested that narratives from the Mass Observation Project Dementia Directive afford a unique perspective to supplement the existing literature concerning the level of public understanding and awareness about dementia in the UK.

\section{Acknowledgements}

The authors wish to express their extreme gratitude to all the respondents who took part in the Spring 2015 Mass Observation Project Dementia Directive.

This work was supported by the University of Chester under the QR Grant [QR304].

The Author(s) declare that there is no conflict of interest. The author(s) received no financial support for the research, authorship, and/or publication of this article.

\section{References}

Anderson, L. A., Day, K. L., Beard, R. L., Reed, P. S., \& Wu, B. (2009). The Public's Perceptions about Cognitive Health and Alzheimer's Disease among the US Population: A National Review. Gerontologist, 49, 3-11.

Arai, Y., Arai, A., \& Zarit, S. (2008). What do we know about dementia? A survey on knowledge about dementia in the general public of Japan. International Journal of Geriatric Psychiatry, 23, 433438.

Benbow, S., \& Kingston, P. (2016). 'Talking about my experiences...at times disturbing yet positive': Producing narratives with people living with dementia. Dementia, 15(5), 1034-1052.

Betts Adams, K., \& McClendon, M. (2006). Early stage cognitive impariment: A social work practice and research agenda. Families in Society, 87(4), 590-600.

Bowling, A., \& Gabriel, Z. (2007). Lay theories of quality fo life in older age. Ageing and Society, 27, 827-848.

Cahill, S., Pierce, M., Werner, P., Darley, A., \& Bobersky, A. (2015). A systematic review of the public's knowledge and understanding of Alzheimer's disease and dementia. Alzheimer Disease Assoc Disord, 29, 255-275.

Carpenter, B. D., Balsis, S., Otilingam, P. G., Hanson, P. K., \& Gatz, M. (2009). The Alzheimer's Disease Knowledge Scale: Development and Psychometric Properties. The Gerontologist, 49(2), 236247.

Chung, J. C. (2000). Lay interpretation of dementia. Int Psychogeriatr, 12, 369-377.

Dementia Strategy (UK) 2017-2020. Dementia UK strategy 2017-2020. Retrieved from London

Dieckmann, L., Zarit, S. H., Zarit, J. M., \& Gatz, M. (1988). The Alzheimer's disease knowledge test. The Gerontologist, 28, 402-407.

Dwyer, C. (Writer) \& National Public Radio (Director). (2018). Pfizer halts research into Alzheimer's and Parkinson's treatments., The Two Way - Breaking News, . USA,.

Gethin-Jones, S. (2014). Familial perceptions of the impact of outcome-focused homecare with older people experiencing dementia and living alone. Working with Older People, 18(2), 90-96. 
Giebel, C. M., Sutcliffe, C., \& Challis, D. (2015). Activities of daily living and quality of life across different stages of dementia: a UK study. Aging and Mental Health, 19(1), 63-71.

Kenigsberg, P. A., Aquino, J. P., Berard, A., Gzil, F., Andrieu, S., Banerjee, S., . . Robert, P. (2016). Dementia beyond 2025: Knowledge and uncertainties. Dementia, 15(1), 6-21. doi:10.1177/1471301215574785

Kim, S., Sargent-Cox, K. A., \& Anstey, K. J. (2015). A qualitative study of older and middle-aged adults' peception and attitudes towards dementia and dementia risk reduction. Journal of Adult Nursing, 71(7), 1694-1703.

Knopman, D. S., DeKosky, S. T., Cummings, J. L., Chui, H., Corey-Bloom, J., Relkin, N., . . Stevens, J. C. (2001). Practice Parameter: Diagnosis of Dementia (an evidence-based review). American Academy of Neurology, 56, 1143-1153.

Langdon, S. A., Eagle, A., \& Warner, J. (2007). Making sense of dementia in the social world: A qualitative study. Social Science and Medicine, 64, 989-1000.

Live in Care Hub. (2017). Dementia overtakes cancer as UK's most feared illness. Retrieved from http://www.liveincarehub.co.uk/dementia-overtakes-cancer-uks-feared-illness/

Milne, A. (2010). The ' $D$ ' word: Reflections on the relationship between stigma, discrimination and dementia. Journal of Mental Health, 19(3), 227-233.

Nielsen, T. R., \& Waldemar, G. (2016). Knowledge and perceptions of dementia and Alxheimer's disease in four ethnic groups in Copenhagen, Denmark. Int J Geriatr Psychiatry, 31(3), 222230.

Organisation. (2012). Dementia - A public health priority. Retrieved from United Kingdom: http://apps.who.int/iris/bitstream/10665/75263/1/9789241564458 eng.pdf?ua=1

Parkinson, M., Carr, S. M., Rushmer, R., \& Abley, C. (2016). Investigating what works to support family carers of people with dementia: a rapid realist review. Journal of Public Health, 1-12. doi:https://doi.org/10.1093/pubmed/fdw100

Parveen, S., Peltier, C., \& Oyebode, J. R. (2017). Perceptions of dementia and use of services in minority ethnic communities: a scoping exercise. Health and Social Care in the Community, 25(2), 734742.

QSR International Pty Ltd. (2017). NVivo 11 Pro qualitative data analysis software, Version 11,. Sydney, Australia.

Quince, C. (2012). Home Truths: Housing services and support for people with dementia. Retrieved from www.alzheimers.org.uk/hometruths

Rabins, P. V. (1998). The caregiver's role in Alzheimer's disease. Dement Geriatr Cogn Disord, 9, 5-28.

Rimmer, E., Wojciechowska, M., \& Stave, C. (2005). Implications fo the Facing Dementia Survey for the general population, patients and caregivers across Europe. Int Journal of Clin Pract Suppl., 59, 17-24.

Roberts, J., McLaughlin, S., \& Connell, C. (2014). Public beliefs and knowledge about risk and protective factors for Alzheimer's Disease. Alzheimer's and Dementia, 10, S381-389.

Steckenrider, J. S. (1993). What people know about Alzheimer's disease: A study of public knowledge. American Journal of Alzheimer's Disease and Other Dementias, 8(1), 6/14.

Stephan, B., \& Brayne, C. (2010). Prevelance and projections of dementia. In M. Downs \& B. Bowers (Eds.), Excellence in Dementia care: Research into Practice (pp. 9-34). Berkshire: Open University Press.

Stokes, L., Combes, H., \& Stokes, G. (2015). The dementia diagnosis: a literature review of information, understanding and attributions. Psychogeriatrics, 15, 218-225.

Sullivan, K. A., Muscat, T. M., \& Mulgrew, K. E. (2007). Knowledge of Alzheimer's Disease amongst patients, carers and non-carer adults: misconceptions, knowledge gaps and correct beliefs. Top Geriatr Rehabil, 23, 148-159.

Van Gorp, B., \& Vercruysse, T. (2012). Frames and counter-frames giving meaning to dementia: a framing analysis of media content. Social Sciences and Medicine, 74(8), 1274-1281. 
Williams, C., Tappen, R., Rosselli, M., Keane, F., \& Newlin, K. (2010). Willingness to be screened and tested for cognitive impariment: cross-culture comparison. American Journal of Alzheimer's Disease and Other Dementias, 25, 160-166.

Yeo, L. H. J., Horan, M. A., Jones, M., \& Pendleton, N. (2007). Perceptions of risk and prevention of dementia in the healthy elderly. Dementia and Geriatric Cognitive Disorders, 23, 368-371.

Zarit, S. H., Gaugler, J. E., \& Jarrott, S. E. (1999). Useful services for families: research findings and direction. Int J Geriatr Psychiatry, 14, 165-178.

Zeilig, H. (2015). What do we mean when we talk about dementia? Exploring cultural representation of 'dementia'. Working with Older People, 19(1), 12-20. 